\title{
Non-radial modes in AGB stars
}

\author{
Josefina Montalbán ${ }^{1,3, \star}$, Michele Trabucchi ${ }^{1}$, Paola Marigo ${ }^{1}$, Peter R. Wood ${ }^{2}$, and Giada \\ Pastorelli $^{1}$ \\ ${ }^{1}$ Department of Physics and Astronomy "Galileo Galilei", University of Padua, Vicolo dell'Osservatorio 3, \\ I-35122 Padova, Italy \\ ${ }^{2}$ Research School of Astronomy and Astrophysics, Australian National University, Canberra, ACT2611, \\ Australia \\ ${ }^{3}$ Observatory of Padua-INAF, Vicolo dell'Osservatorio 5, I-35122 Padova, Italy
}

\begin{abstract}
The success of asteroseismology in characterising G-K giants has motivated the extension of the same techniques to stars after the central He-burning and M-giants. The latter have been usually studied only as radial pulsators; the presence, however, of fine-structure in the period-luminosity diagram of red variables in the Magellanic Clouds could result from the presence of non-radial oscillations, offering the potential of observational indexes based on non-radial oscillations also for luminous red giants. We present here the results of a first approach aiming to identify the origin of the sub-ridges in the sequence $\mathrm{A}$ of the LMC red variables.
\end{abstract}

\section{Method and results}

After the central H- and He-burning, the density contrast between the core and the envelope increases with luminosity, and these stellar regions become dynamically decoupled. Radial and non-radial modes are then strongly trapped in the envelope, and their oscillation frequencies are determined only by the physical properties of that region. This hypothesis is generally accepted for radial and quadrupole modes, but it does not always hold for dipole ones. We have verified its validity for dipole modes $(\ell=1)$ in $1.5 \mathrm{M}_{\odot}$ AGB models, by computing $\ell=1$ frequency oscillations for complete and envelope models. These results are consistent with those by [3] for luminous RGB stars.

Using the adiabatic oscillation code LOSC ([2]), we have computed radial and non-radial $(\ell=1,2)$ oscillation frequencies for AGB envelope models ([1]) for three masses $\left(0.8,1.5\right.$ and $\left.2.0 \mathrm{M}_{\odot}\right)$ with a significant representation in the LMC population synthesis. The left panel of Figure 1 shows the period-luminosity (PL) diagram for a luminosity sequence of a $1.5 \mathrm{M}_{\odot}$ envelope model. Adiabatic computations do not allow to identify the dominant mode (P1), hence, we have considered all the possible theoretical period-pairs from the 6 modes closest to $P_{\max }$ (period at maximum emission in the framework of stochastic oscillations). The results are shown in the right panel of Figure 1, a Petersen diagram where we plot the period ratios $\log (P 2 / P 1)$ and $\log (P 3 / P 1)(P 1, P 2$ and $P 3$ are the three observed periods for each star - black dots - in the LMC, with P1 being the dominant one) against $\log P 1\left[W_{J K}=12\right]$ (the value of $\log P 1$ projected along sequence $\mathrm{A}$, see [4] for details).

^ josefa.montalbaniglesias@unipd.it 

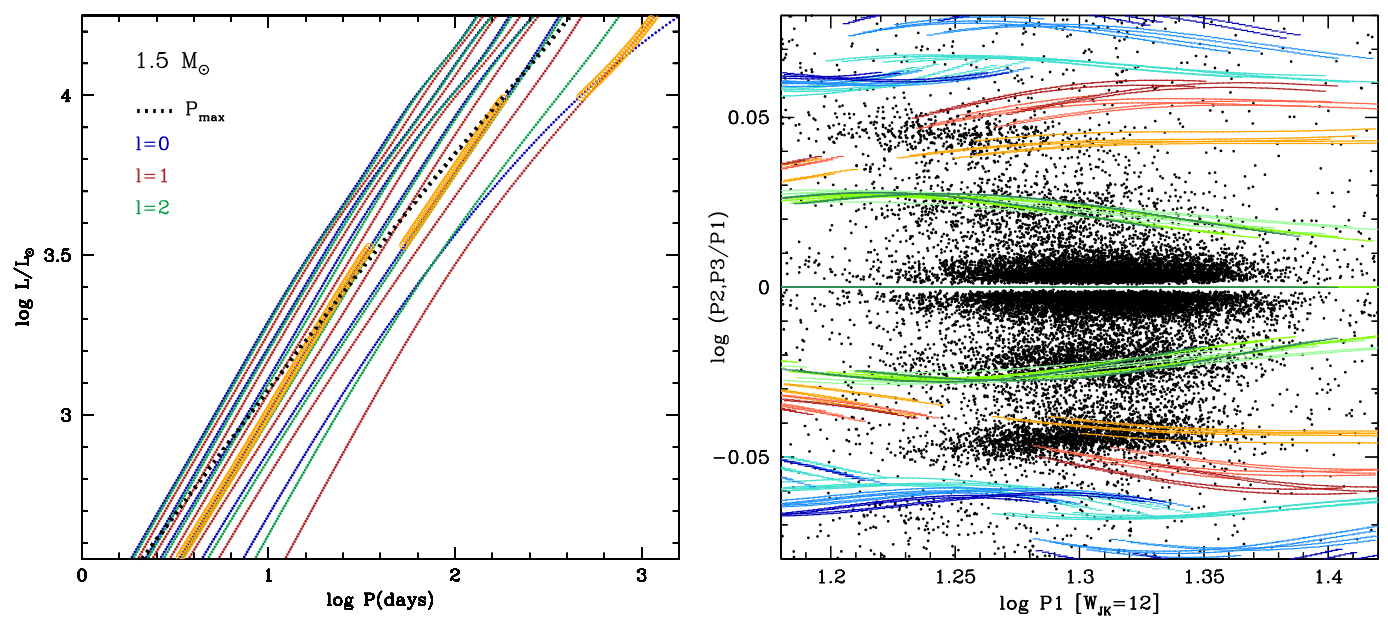

Figure 1. Left panel: Luminosity versus period for radial and non-radial modes (dipole and quadrupole) of $1.5 \mathrm{M}_{\odot}$ envelope models along a luminosity sequence. Different lines correspond to fundamental and five consecutive overtones. Dotted black line is the PL relation corresponding to $v_{\max }$ from scaling relation ([6]). Orange symbols indicate the dominant modes as those with the largest growth rate given by the non-adiabatic radial computation. Right panel: Petersen diagram as that in Figure 3 of [4]. Black dots correspond to period ratios from LMC observations ([5]), and coloured ones to theoretical values obtained from luminosity sequences for 0.8, 1.5 and $2.0 \mathrm{M}_{\odot}$, LMC metallicity, O- and C-rich chemical composition. Different colours corresponds to different pairs of angular degrees involved in the period ratio: $\ell=0$ and $\ell=1$ modes, blue dots; $\ell=1$ and $\ell=2$, red-orange ones; $\ell=0$ and $\ell=2$, green ones. The lightest colours are for the highest mass, and the darkest ones for lowest mass.

While the sub-ridges at $\log (P 2 / P 1) \sim \pm 0.05$ seem defined by the periods of $\ell=1$ and $\ell=2$ modes and their characterisation should require more detailed modelling, the sub-ridges at $\log (P 2 / P 1) \sim$ \pm 0.02 are completely determined by the period difference between $\ell=0$ and $\ell=2$ modes, and their location does not depend on total stellar mass, He-core mass, or chemical composition (O- or C-rich models).

Acknowledgments: We acknowledge the support from the ERC Consolidator Grant funding scheme (project STARKEY, G.A. n. 615604).

\section{References}

[1] Fox, M., \& Wood, P. R., ApJ, 259,198 (1982)

[2] Scuflaire, R., Montalbán, J., Théado, S., Bourge, P.-O., Miglio, A., Godart, M., Thoul, A., \& Noels, A., Ap\&SS, 316, 149 (2008)

[3] Dziembowski, W. A., A\&A, 539, A83 (2012)

[4] Wood, P. R., MNRAS, 448, 3829 (2015)

[5] Soszyński, I., et al., Acta Astron., 59, 239 (2009)

[6] Kjeldsen, H., \& Bedding, T. R., A\&A, 293, 87 (1995) 